\title{
On Condition for Output Finite-Time Stability and Adaptive Finite-Time Control Scheme*
}

\author{
Konstantin Zimenko ${ }^{\dagger}$, Denis Efimov ${ }^{\ddagger}, \dagger$ and Andrey Polyakov ${ }^{\ddagger, \dagger}$
}

\begin{abstract}
A sufficient condition for output finite-time stability is presented. Based on this condition a scheme of adaptive finite-time control design is provided. The presented results are obtained with the use of homogeneity property. The theoretical results are supported by numerical examples.
\end{abstract}

\section{INTRODUCTION}

Frequently, the control practice needs regulation algorithms, which ensure output (in particular, a part of states) convergence in a finite time (i.e. the output $y\left(t, x_{0}\right)=0$ for all $t \geq T_{0}$ and some $\left.0 \leq T_{0}<+\infty\right)$. Such problem statements usually appear in mechanical and robotic systems, aerospace applications, particle collision systems (see, for example, [1], [2], [3], [18], etc.).

In addition, certain classes of identification problems and adaptive control systems may be considered in the context of output stability. For example, in the case of adaptive control design a plant with an obtained feedback has a state vector extended with adjustable control parameters, i.e. $\left[x^{T} \omega^{T}\right]^{T}$, where $x \in \mathbb{R}^{n}$ is the state vector of the plant and $\omega \in \mathbb{R}^{r}$ is the adjustable control parameters vector. Thus, the main problem is to guarantee output (partial) stability: the states of the plant should be stabilized, while the adjustable parameters' dynamics may remain unstable.

There are a number of results devoted to output finite-time stability (OFTS) analysis. Most of them are about partial stability analysis that is a particular case of output stability (see, for example, [18], [3], [4]). In [11] necessary and sufficient conditions for output finite-time stability are given using Lyapunov functions. However, most of these results are obtained for rather restricted class of systems, and/or they are difficult to apply.

The present paper provides sufficient condition to ensure OFTS. The results presented in this paper are related to the wider class then in [11]. The result on partial stability [18] is a particular case of the condition. Further, based on the provided condition, a scheme of adaptive finite-time control design is presented.

The paper is organized in the following way. Notation used in the paper is given in Section II. Section III recalls basics on output stability (OS), OFTS and homogeneity property.

*This work is supported by the Russian Science Foundation under grant 17-19-01422.

${ }^{\dagger}$ Konstantin Zimenko, Andrey Polyakov and Denis Efimov are with Faculty of Control Systems and Robotics, ITMO University, 49 Kronverkskiy av., 197101 Saint Petersburg, Russia.

${ }^{\ddagger}$ Andrey Polyakov and Denis Efimov are with Inria, Univ. Lille, CNRS, UMR 9189 - CRIStAL, F-59000 Lille, France.

e-mail: kostyazimenko@gmail.com,

andrey.polyakov@inria.fr, denis.efimoveinria.fr
Section IV presents the main result on sufficient condition of OFTS and adaptive finite-time control design with numerical examples. Finally, concluding remarks are given in Section $\mathrm{V}$.

\section{NOTATION}

Through the paper the following notation are used:

- $\mathbb{R}^{n}$ denotes the $n$ dimensional Euclidean space with vector norm $|\cdot|$;

- $\mathbb{R}_{+}=\{x \in \mathbb{R}: x>0\}$, where $\mathbb{R}$ is the field of real numbers;

- The symbol $\overline{1, m}$ is used to denote a sequence of integers $1, \ldots, m$;

- a continuous function $\sigma: \mathbb{R}_{+} \cup\{0\} \rightarrow \mathbb{R}_{+} \cup\{0\}$ belongs to class $\mathcal{K}$ if it is strictly increasing and $\sigma(0)=0$. It belongs to class $\mathcal{K}_{\infty}$ if it is also radially unbounded;

- A continuous function $\beta: \mathbb{R}_{+} \cup\{0\} \times \mathbb{R}_{+} \cup\{0\} \rightarrow$ $\mathbb{R}_{+} \cup\{0\}$ belongs to class $\mathcal{K} \mathcal{L}$ if $\beta(\cdot, r) \in \mathcal{K}$ and $\beta(r, \cdot)$ is decreasing to zero for any fixed $r \in \mathbb{R}_{+}$.

\section{PRELIMINARIES}

Consider the system in the form

$$
\dot{x}=f(x), \quad y=h(x)
$$

with states $x \in \mathbb{R}^{n}$ and outputs $y \in \mathbb{R}^{p}$. Let the system satisfy the following assumptions:

(A.1) The vector field $f: \mathbb{R}^{n} \rightarrow \mathbb{R}^{n}$ ensures forward existence and uniqueness of the system solutions at least locally in time, $f(0)=0$.

(A.2) The function $h: \mathbb{R}^{n} \rightarrow \mathbb{R}^{p}$ is continuously differentiable, $h(0)=0$ and there exists $\gamma \in \mathcal{K}$ such that $|h(x)| \leq \gamma(|x|)$ for all $x \in \mathbb{R}^{n}$.

(A.3) Let the vector field $f \in \mathcal{C}\left(\mathbb{R}^{n}\right)$ be locally Lipschitz continuous on $\mathbb{R}^{n} \backslash \mathcal{Y}$, where $\mathcal{Y}=\left\{x \in \mathbb{R}^{n}: h(x)=0\right\}$.

For the initial conditions $x_{0} \in \mathbb{R}^{n}$, let $\Phi\left(t, x_{0}\right)$ be a unique solution of the system (1) defined over a finite interval $\left[0, T_{s}\right)$ with some $T_{s}>0, y\left(t, x_{0}\right)=h\left(\Phi\left(t, x_{0}\right)\right)$.

Note that the preliminaries in this subsection are based on theoretical framework of Input-to-Output Stability and uniform output stability (UOS) presented for locally Lipschitz continuous systems in [5]-[10]. In [11] the results on UOS were extended for wider class, where the Lipschitz continuity may be violated on $\mathcal{Y}$ (i.e. the assumption A.3 is satisfied).

Definition 1 [6], [7] A system is forward complete if each $x_{0} \in \mathbb{R}^{n}$ produces a solution $\Phi\left(t, x_{0}\right)$ which is defined on $[0, \infty)$. 
Definition 2 [5] The system (1) has the unboundedness observability $(U O)$ property if

$$
\limsup _{t \rightarrow T_{s}}\left|y\left(t, x_{0}\right)\right|=+\infty
$$

necessarily follows for each $x_{0} \in \mathbb{R}^{n}$ with $T_{s}<+\infty$.

In other words, any unboundedness of the state vector can be observed using the output $y$. Hence, if the output is known to be bounded (which is the case under the output stability properties described below) then the UO property is equivalent to forward completeness [7]. Note, that any system has the property of UO in the output $h(x)=x$.

Definition 3 [8], [9] A system (1) is output stable (OS) if

- it is forward complete, and

- there exists a $\mathcal{K} \mathcal{L}$-function $\beta$ such that

$$
\left|y\left(t, x_{0}\right)\right| \leq \beta\left(\left|x_{0}\right|, t\right) \quad \forall t \geq 0
$$

holds for all $x_{0} \in \mathbb{R}^{n}$.

If, in addition, there exists $\sigma \in \mathcal{K}$ such that

$$
\left|y\left(t, x_{0}\right)\right| \leq \sigma\left(\left|h\left(x_{0}\right)\right|\right) \quad \forall t \geq 0
$$

holds for all trajectories of the system, then the system is output-Lagrange output stable (OLOS). Finally, if one strengthens (2) to

$$
\left|y\left(t, x_{0}\right)\right| \leq \beta\left(\left|h\left(x_{0}\right)\right|, t\right), \quad \forall t \geq 0
$$

holding for all trajectories of the system, then the system is state-independent output stable (SIOS).

Lemma 1 [10] For system (1) having the UO property, the following relations are valid:

$$
S I O S \Rightarrow O L O S \Rightarrow O S .
$$

In the general case, all inverse relations are not satisfied.

Let us present definitions for corresponded Lyapunov functions.

Definition 4 [6], [7] For the system (1), a smooth function $V$ and a function $\lambda: \mathbb{R}^{n} \rightarrow \mathbb{R}_{+} \cup\{0\}$ are called respectively an OS-Lyapunov function and an auxiliary modulus if there exist $\alpha_{1}, \alpha_{2} \in \mathcal{K}_{\infty}$ so that

$$
\alpha_{1}(|h(x)|) \leq V(x) \leq \alpha_{2}(|x|) \quad \forall x \in \mathbb{R}^{n}
$$

holds and there exists $\alpha_{3} \in \mathcal{K} \mathcal{L}$ such that

$$
\dot{V}(x) \leq-\alpha_{3}(V(x), \lambda(x))
$$

for all $x \in \mathcal{X}$, where $\mathcal{X}=\left\{x \in \mathbb{R}^{n}: V(x)>0\right\}$, and either

(a) $\lambda$ satisfies the following conditions:

- $0 \leq \lambda(x) \leq|x|$ for all $x \in \mathbb{R}^{n}$;

- $\lambda$ is locally Lipschitz on the set $\mathcal{X}$ and satisfies

$$
D \lambda(x) f(x) \leq 0
$$

for almost all $x \in \mathcal{X}$,

or

(b) there exists some $\theta \in \mathcal{K}$ such that

$$
\lambda\left(\Phi\left(t, x_{0}\right)\right) \leq \theta\left(\left|x_{0}\right|\right)
$$

for all $t \geq 0$ and $x \in \mathcal{X}$.
The function $V$ is called an OLOS-Lyapunov function if it is an OS-Lyapunov function, and in addition, inequality (5) can be strengthened to

$$
\alpha_{1}(|h(x)|) \leq V(x) \leq \alpha_{2}(|h(x)|), \quad \forall x \in \mathbb{R}^{n} .
$$

The function $V$ is called the SIOS-Lyapunov function if the inequality (9) is satisfied and there exists $\alpha_{3} \in \mathcal{K}$ such that for all $x \in \mathcal{X}$ :

$$
\dot{V}(x) \leq-\alpha_{3}(V(x))
$$

An auxiliary modulus $\lambda$ satisfying property (a) is called a strong auxiliary modulus, and one satisfying property (b) is a weak auxiliary modulus [7].

Note that in the case of OLOS- or SIOS-Lyapunov function we have $\mathcal{X}=\mathbb{R}^{n} \backslash \mathcal{Y}$.

Remark 1 In [5]-[10] all given above definitions are presented in the sense of uniform stability with respect to inputs $u$ for the system $\dot{x}=f(x, u), y=h(x)$.

The following theorem gives the necessary and sufficient Lyapunov characterizations of output stability for the system (1).

Theorem 1 [11] Suppose the system (1) is UO.

(1) The following claims are equivalent for the system:

(a) it is OLOS;

(b) it admits an OLOS-Lyapunov function with a weak auxiliary modulus;

(c) it admits an OLOS-Lyapunov function with a strong auxiliary modulus.

(2) The following claims are equivalent for the system:

(a) it is SIOS;

(b) it admits an SIOS-Lyapunov function.

\section{A. Output Finite-Time Stability}

Let us present the definition on output finite-time stability.

Definition 5 The system (1) is said to be OFTS if it is OS and for any $x_{0} \in \mathbb{R}^{n}$ there exists $0 \leq T_{0}<+\infty$ such that $y\left(t, x_{0}\right)=0$ for all $t>T_{0}$. The function $T\left(x_{0}\right)=\inf \left\{T_{0} \geq\right.$ $\left.0: y\left(t, x_{0}\right)=0 \forall t \geq T_{0}\right\}$ is called the settling-time function.

Definition 6 The system (1) is said to be output fixed-time stable if it is OFTS and $\sup T\left(x_{0}\right)<+\infty$.

The paper [18] deals with partial finite-time stability that is a particular case of OFTS, where $h$ is a projection on a subspace of the state space $\mathbb{R}^{n}$.

Theorem 2 [18] Consider the system

$$
\begin{array}{ll}
\dot{x}_{1}=f_{1}\left(x_{1}, x_{2}\right), & x_{1}(0)=x_{10}, \\
\dot{x}_{2}=f_{2}\left(x_{1}, x_{2}\right), & x_{2}(0)=x_{20},
\end{array}
$$

where $x_{1} \in \mathcal{N} \subseteq \mathbb{R}^{n_{1}}, x_{2} \in \mathbb{R}^{n_{2}}$ are the states, $f_{1}$ : $\mathcal{N} \times \mathbb{R}^{n_{2}} \rightarrow \mathbb{R}^{n_{1}}$ and $f_{2}: \mathcal{N} \times \mathbb{R}^{n_{2}} \rightarrow \mathbb{R}^{n_{2}}$ are such that, for every $\left(x_{1}, x_{2}\right) \in \mathcal{N} \times \mathbb{R}^{n_{2}}, f_{1}\left(0, x_{2}\right)=0$ and $f_{1}(\cdot, \cdot)$, $f_{2}(\cdot, \cdot)$ are jointly continuous in $x_{1}$ and $x_{2}$. If there exist a continuously differentiable function $V: \mathcal{N} \times \mathbb{R}^{n_{2}} \rightarrow \mathbb{R}$, a

\footnotetext{
${ }^{1}$ If $X, Y$ and $Z$ are topological spaces and $f: X \times Y \rightarrow Z$ is a function then we say that $f$ is jointly continuous at $\left(x_{0}, y_{0}\right) \in X \times Y$ if for each neighbourhood $W$ of $f\left(x_{0}, y_{0}\right)$ there exists a product of open sets $U \times V \subseteq X \times Y$ containing $\left(x_{0}, y_{0}\right)$ such that $f(U \times V) \subseteq W$.
} 
class $\mathcal{K}$ function $\alpha(\cdot)$, a continuous function $k:[0,+\infty) \rightarrow$ $\mathbb{R}_{+}$, a real number $\mu \in(0,1)$ and an open neighbourhood $\mathcal{M} \subseteq \mathcal{N}$ of $x_{1}=0$ such that for $\left(x_{1}, x_{2}\right) \in \mathcal{M} \times \mathbb{R}^{n_{2}}$

$$
\begin{gathered}
V\left(0, x_{2}\right)=0 \\
\alpha\left(\left|x_{1}\right|\right) \leq V\left(x_{1}, x_{2}\right), \\
\dot{V}\left(x_{1}, x_{2}\right) \leq-k\left(\left|x_{2}\right|\right)\left(V\left(x_{1}, x_{2}\right)\right)^{\mu}
\end{gathered}
$$

then (11) is finite-time stable with respect to $x_{1}$ (OFTS with $\left.y=x_{1}\right)$. Moreover, there exist a neighbourhood $\mathcal{N}_{0}$ of $x_{1}=0$ and a settling-time function $T: \mathcal{N}_{0} \times \mathbb{R}^{n_{2}} \rightarrow[0, \infty)$ such that

$$
T\left(x_{10}, x_{20}\right) \leq q^{-1}\left(\frac{\left(V\left(x_{10}, x_{20}\right)\right)^{1-\mu}}{1-\mu}\right),\left(x_{10}, x_{20}\right) \in \mathcal{N}_{0} \times \mathbb{R}^{n_{2}},
$$

where $q:[0, \infty) \rightarrow \mathbb{R}$ is continuously differentiable and satisfies

$$
\dot{q}(t)=k\left(\left|x_{2}(t)\right|\right), \quad q(0)=0, \quad t \geq 0,
$$

and $T(\cdot, \cdot)$ is jointly continuous on $\mathcal{N}_{0} \times \mathbb{R}^{n_{2}}$.

In the paper [11] necessary and sufficient Lyapunov characterizations of output finite-time stability are presented for the class of OLOS and SIOS systems (1). The following corollary on OFTS property is used in the paper.

Corollary 1 [11] Consider the SIOS system (1). Let a SIOS-Lyapunov function satisfies the inequality

$$
\dot{V}(x) \leq-c V(x)^{\mu}
$$

for some $c \in \mathbb{R}_{+}, \mu \in(0,1)$ and all $x \in \mathcal{X}$. Then the system (1) is OFTS. Moreover, the settling-time function satisfies

$$
T(x) \leq \frac{1}{c(1-\mu)} V(x)^{1-\mu} .
$$

\section{B. Homogeneity}

Homogeneity [15] is an intrinsic property of an object, which remains consistent with respect to some scaling. This property provides many advantages to analysis (including finite-time stability analysis) and design of nonlinear control system.

For $r_{i} \in \mathbb{R}_{+}, i=\overline{1, n}, \rho \in \mathbb{R}_{+}$and $\lambda>0$ define vector of weights $r=\left(\begin{array}{lll}r_{1}, & \ldots, & r_{n}\end{array}\right)^{T}$, dilation matrix $D_{r}(\lambda)=$ $\operatorname{diag}\left\{\lambda^{r_{i}}\right\}_{i=1}^{n}$ and homogeneous norm

$$
\|x\|_{r}=\left(\sum_{i=1}^{n}\left|x_{i}\right|^{\frac{\rho}{r_{i}}}\right)^{\frac{1}{\rho}} .
$$

Definition 7 [15] A function $g: \mathbb{R}^{n} \rightarrow \mathbb{R}$ (vector field $f: \mathbb{R}^{n} \rightarrow \mathbb{R}^{n}$ ) is said to be r-homogeneous of degree $d$ if

$$
\begin{gathered}
g\left(D_{r}(\lambda) x\right)=\lambda^{d} g(x) \\
\left(f\left(D_{r}(\lambda) x\right)=\lambda^{d} D_{r}(\lambda) f(x)\right)
\end{gathered}
$$

for fixed $r$, all $\lambda>0$ and $x \in \mathbb{R}^{n}$.

Introduce the following compact set (homogeneous sphere) $\mathbb{S}_{r}=\left\{x \in \mathbb{R}^{n}:\|x\|_{r}=1\right\}$, then for any $x \in \mathbb{R}^{n}$ there is $y \in \mathbb{S}_{r}$ such that $x=D_{r}(\lambda) y$ for $\lambda=\|x\|_{r}$.
Theorem 3 [16], [17] Let $f: \mathbb{R}^{n} \rightarrow \mathbb{R}^{n}$ be defined on $\mathbb{R}^{n}$ and be a continuous $r$-homogeneous vector field with degree $\nu(\nu<0)$. If the origin of the system $\dot{x}=f(x)$ is locally asymptotically stable then it is globally asymptotically stable (globally finite-time stable) and there exists a continuously differentiable Lyapunov function $V$ which is $r$-homogeneous with the degree $\mu>-\nu$.

According to [15], [17] there exist constants $c_{1}, c_{2} \in \mathbb{R}_{+}$, such that

$$
c_{1}\|x\|_{r}^{\mu} \leq V(x) \leq c_{2}\|x\|_{r}^{\mu}
$$

and

$$
\frac{\partial V(y)}{\partial y} f(y) \leq-\bar{a}, \quad\left|\frac{\partial V(y)}{\partial y}\right| \leq b \quad \forall y \in \mathbb{S},
$$

where $\bar{a}, b \in \mathbb{R}_{+}$.

A nonlinear system $\dot{x}=f(x, u)$ is homogeneously stabilizable with degree $\nu \in \mathbb{R}$ if there exists a feedback $u(x)$ such that the closed-loop system is homogeneous of degree $\nu$ and globally asymptotically stable. In this case the feedback $u(x)$ is called homogenizing of degree $\nu$.

\section{MAIN RESULTS}

\section{A. On Sufficient Condition for Output Finite-Time Stability}

Consider the system in the form

$$
\dot{x}=f(x), \quad y=h(x)
$$

where $x \in \mathbb{R}^{n}$ is state vector, $y \in \mathbb{R}^{p}$ is output, the vector field $f: \mathbb{R}^{n} \rightarrow \mathbb{R}^{n}$ ensures forward existence and uniqueness of the system solutions at least locally in time, the function $h: \mathbb{R}^{n} \rightarrow \mathbb{R}^{p}$ is continuous, $f(0)=0$ and $h(0)=0$.

Note that the system (19) is of wider class then considered in the preliminaries.

The following theorem provides a sufficient condition for output finite-time stability of the system (19).

Theorem 4 Let there exist positive definite $\mathcal{C}^{1}$ functions $U: \mathbb{R}^{n} \rightarrow \mathbb{R}_{+} \cup\{0\}$ and $W: \mathbb{R}^{n} \rightarrow \mathbb{R}_{+} \cup\{0\}$ such that for $\alpha_{1}, \alpha_{2} \in \mathcal{K}_{\infty}$ the following conditions are satisfied

$$
\begin{gathered}
\alpha_{1}(|h(x)|) \leq U(x) \leq \alpha_{2}(|h(x)|), \\
V(x)=U(x)+W(x), \\
\dot{V}(x) \leq-a U^{\alpha}(x), \\
\dot{V} \leq 0 \Rightarrow|\dot{W}| \leq \sum_{i=1}^{N} b_{i} U^{\beta_{i}}
\end{gathered}
$$

where $a, b_{i} \in \mathbb{R}_{+}, \beta_{i}>\alpha, \alpha \in(0,1), N \in \mathbb{N}$. Then the system (19) is OFTS provided that it is UO.

Remark 2 In general, none of the functions $U(x), W(x)$, $V(x)$ is an output Lyapunov function. However, with respect to Definition 4 we have:

- if $W(x) \leq \alpha_{3}(|h(x)|)$ for $\alpha_{3} \in \mathcal{K}$, then $V(x)$ is SIOSLyapunov function; 
- if $\dot{W}(x) \geq 0$, then $U(x)$ is SIOS-Lyapunov function and the settling-time function is bounded as follows:

$$
T\left(x_{0}\right) \leq \frac{U_{0}^{1-\alpha}}{a(1-\alpha)},
$$

where $U_{0}=U\left(x_{0}\right)$.

\section{Example 1}

Consider the system

$$
\begin{aligned}
& \dot{x}_{1}=-\operatorname{sign}\left(x_{1}\right)\left|x_{1}\right|^{0.5}+x_{1}^{3} \sin \left(x_{2}\right), \\
& \dot{x}_{2}=\left|x_{1}\right|^{3.5}, \\
& y=x_{1} .
\end{aligned}
$$

Clearly the system admits UO property (the right-hand side is bounded for a bounded value of y). For $U(x)=\left|x_{1}\right|^{1.5}$ and $W(x)=1.5\left(1+\cos \left(x_{2}\right)\right)$ the conditions (20)-(23) are satisfied with $\dot{V}(x)=-1.5 U^{2 / 3}$ and $|\dot{W}| \leq 1.5 U^{7 / 3}$. Then the system is OFTS.

\section{B. Adaptive Control Design}

The presented result can be utilized for adaptive finite-time control design. Consider the system

$$
\dot{x}(t)=f(x(t), u(t), \theta, t), \quad x(0)=x_{0}, \quad t \geq 0,
$$

where $x \in \mathbb{R}^{n}$ is the measurable state vector, $u \in \mathbb{R}^{m}$ is the control input, $\theta \in \mathbb{R}^{r}$ is the vector of unknown parameters and $f: \mathbb{R}^{n} \times \mathbb{R}^{m} \times \mathbb{R}^{r} \times \mathbb{R}_{+} \rightarrow \mathbb{R}^{n}$. An adaptive control for stabilizing the system (24) can be presented in the form [19]

$$
\begin{aligned}
& u(t)=g(x(t), \omega(t), t), \\
& \dot{\omega}(t)=\kappa(x(t), t),
\end{aligned}
$$

where $\omega \in \mathbb{R}^{r}$ is the adjustable control parameter vector, $g$ and $\kappa$ are mappings defined as $g: \mathbb{R}^{n} \times \mathbb{R}^{r} \times \mathbb{R}_{+} \rightarrow \mathbb{R}^{m}$ and $\kappa: \mathbb{R}^{n} \times \mathbb{R}_{+} \rightarrow \mathbb{R}^{r}$. Then the problem of adaptive control design is to provide output finite-time stability (finite-time partial stability) of the system (24), (25) with the output $y=$ $x$ and extended state vector $\left[x^{T} \omega^{T}\right]^{T}$ (i.e. we are interested only in convergence of $x$ at the origin).

To demonstrate how the result of Theorem 4 can be utilized for adaptive finite-time control design let us consider the system (24) in the form

$$
\dot{x}=A x+B\left(\phi(x)^{T} \theta+u\right),
$$

where $x \in \mathbb{R}^{n}, u \in \mathbb{R}$, the pair of system matrix $A \in \mathbb{R}^{n \times n}$ and control gain matrix $B \in \mathbb{R}^{n \times 1}$ is controllable and $\phi$ : $\mathbb{R}^{n} \rightarrow \mathbb{R}^{r}$ is known. Then following Theorem 4 one may obtain:

Theorem 5 Let $u_{F T S}: \mathbb{R}^{n} \rightarrow \mathbb{R}$ be homogenizing of degree $\nu<0$ and stabilizing in a finite time control law for the linear system

$$
\dot{x}=A x+B u_{F T S}(x)
$$

and $V_{F T S}(x)$ be the corresponding homogeneous Lyapunov function of degree $\mu$. Let $|\phi(x)|<c\|x\|_{r}^{\delta}$ for some $c \in \mathbb{R}_{+}$ and $\delta>\nu+r_{\max }$. Then with adaptive control in the form

$$
\begin{aligned}
& u(x, \omega)=u_{F T S}(x)+\phi(x)^{T} \omega \\
& \dot{\omega}=\gamma \phi(x)\left(\frac{\partial V_{F T S}(x)}{\partial x} B\right)^{T}
\end{aligned}
$$

for some $\gamma \in \mathbb{R}_{+}$the system (26) is OFTS with respect to the output $y=x$ and the variable $\omega$ stays bounded.

Due to homogeneity property of (27) the presented control scheme implies some robustness abilities of (26) as, for example, it can cancel certain non-Lipschitz disturbances.

\section{Example 2}

Consider the system (26) with

$$
\begin{array}{ll}
A=\left(\begin{array}{lll}
0 & 1 & 0 \\
0 & 0 & 1 \\
0 & 0 & 0
\end{array}\right), & B=\left(\begin{array}{c}
0 \\
0 \\
1
\end{array}\right), \\
\phi(x)=\left(\begin{array}{c}
\sin \left(x_{1}\right) \\
x_{2}
\end{array}\right), & \theta=\left(\begin{array}{c}
3 \\
-1
\end{array}\right) .
\end{array}
$$

According to [20] choose finite-time control law for the system (27) as

$$
u_{F T S}=V_{F T S}^{1-\varrho} k D_{r}\left(V_{F T S}^{-1}\right) x
$$

with

- $\varrho \in(0,1), r=\left(\begin{array}{lll}1+2 \varrho & 1+\varrho & 1\end{array}\right)^{T}$;

- $k=Y X^{-1}$, where $Y \in \mathbb{R}^{3 \times 1}, X \in \mathbb{R}^{3 \times 3}$ is a solution of linear matrix inequalities

$$
\left\{\begin{array}{l}
A X+X A^{T}+B Y+Y^{T} B^{T}>0, \\
X \operatorname{diag}\left\{r_{i}\right\}_{i=1}^{n}+\operatorname{diag}\left\{r_{i}\right\}_{i=1}^{n} X>0, \quad X>0
\end{array}\right.
$$

- $V_{F T S} \in \mathbb{R}_{+}$satisfies $Q\left(V_{F T S}, x\right)=0$, where

$$
Q\left(V_{F T S}, x\right)=x^{T} D_{r}\left(V_{F T S}^{-1}\right) X^{-1} D_{r}\left(V_{F T S}^{-1}\right) x-1 .
$$

Then according to Theorem 5 the system is finite-time stable with the use of adaptive control in the form (28). The results of simulation are shown in Fig. 1. The results of simulation with using the logarithmic scale are shown in Fig. 2 in order to demonstrate finite-time convergence rate of $|x|$. The form of the control $u(x)=u_{F T S}(x)$ is shown in Fig. 3 demonstrating that the control without adaptive term may not guarantee stability of the system.

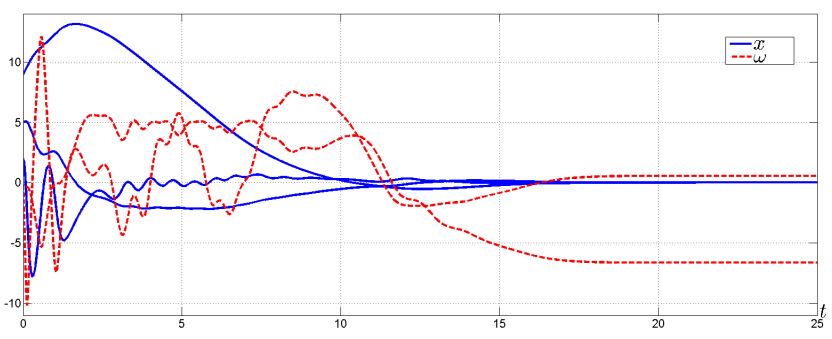

Fig. 1. System states $x, \omega$ versus time

Remark 3 The control scheme (28) presented in Theorem 5 can be applied not necessary with homogenising control laws $u_{F T S}$. Since for finite-time stable system there exists a Lyapunov function that $\dot{V}_{F T S}(x) \leq-a V_{F T S}^{\alpha}(x), a \in$ $\mathbb{R}_{+}, \alpha \in(0,1)$ then the main condition for applying the scheme (28) is

$$
\left|\frac{\partial V_{F T S}(x)}{\partial x} B\right||\phi(x)| \leq \sum_{i=1}^{N} b_{i} U^{\beta_{i}},
$$

where $b_{i} \in \mathbb{R}_{+}, \beta_{i}>\alpha, N \in \mathbb{N}$. 


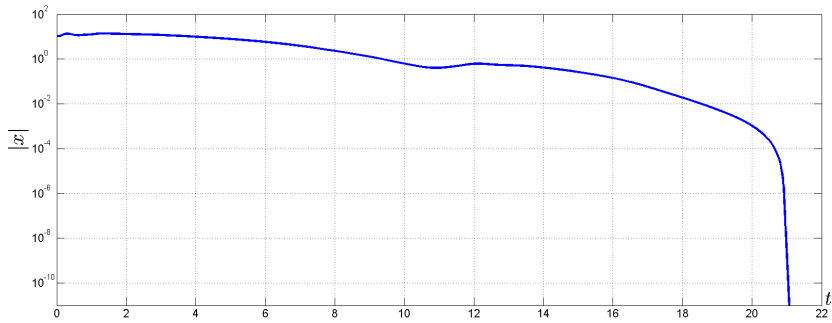

Fig. 2. Simulation plot of $|x|$

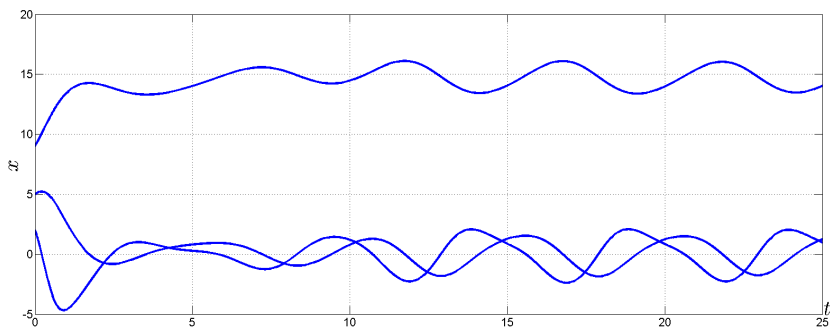

Fig. 3. System states $x$ for the control without adaptive term

\section{CONCLUSIONS}

In the paper a sufficient condition of output finite-time stability is presented. Comparing with analogues researches the presented result is less restrictive and/or obtained for a wider class of systems. Based on the provided sufficient condition, a simple scheme of adaptive finite-time control design is presented. Possible directions for future research include control and observer design based on the use of the presented OFTS condition.

\section{REFERENCES}

[1] V.I. Vorotnikov, Partial Stability and Control, Birkhuser, Boston, MA, 1998.

[2] Z. Hai-Ping, M. Feng-Xiang, On the stability of nonholonomic mechanical systems with respect to partial variables, Appl.Math. Mech, vol. 16(3), pp. 237-245, 1995.

[3] C. Jammazi, A discussion on the Hölder and robust finite-time partial stabilizability of Brockett's integrator, ESAIM: Control, Optimisation and Calculus of Variations, vol. 18, pp. 360-382, 2012.

[4] C. Jammazi, Continuous and discontinuous homogeneous feedbacks finite-time partially stabilizing controllable multichained systems, SIAM Journal on Control and Optimization, vol. 52(1), pp. 520-544, 2014.

[5] D. Angeli, E.D. Sontag, Forward completeness, unboundedness observability, and their Lyapynov characterizations, Systems \& Control Letters, vol. 38, pp. 209-217, 1999.

[6] S.N. Dashkovskiy, D.V. Efimov, E.D. Sontag, Input to State Stability and Allied System Properties, Automation and Remote Control, vol. 72, no. 8, pp. 1579-1614, 2011.

[7] B. Ingalls, Y. Wang, On Input-to-Output Stability for Systems not Uniformly Bounded, Proc. NOLCOS, St. Petersburg, July 2001.

[8] E.D. Sontag, Y. Wang, Lyapunov Characterizations of Input to Output Stability, SIAM J. Control Optim., no. 39(1), pp. 226-249, 2000.

[9] E.D. Sontag, Y. Wang, A Notion of Input to Output Stability, Proc. Eur. Control Conf., Brussels, July 1997, DOI: 10.23919/ECC.1997.7082720.

[10] E.D. Sontag, Y. Wang, Notions of Input to Output Stability, Syst. Control Lett., no. 38(4-5), pp. 235-248, 1999.

[11] K. Zimenko, D. Efimov, A. Polyakov, On Notions of Output FiniteTime Stability, 17th European Control Conference (ECC19), 2019, DOI: $10.23919 /$ ECC.2019.8796039.
[12] S. Bhat and D. Bernstein, Finite-time stability of continuous autonomous systems, SIAM Journal of Control and Optimization, vol. 38(3), pp. 751-766, 2000.

[13] Y. Orlov, Finite Time Stability and Robust Control Synthesis of Uncertain Switched Systems, SIAM Journal of Control and Optimization, vol. 43(4), pp. 1253-1271, 2004

[14] - Finite time stability and stabilization of a class of continuous systems, Journal of Mathematical Analysis and Application, vol. 323(2), pp. 1430-1443, 2006.

[15] V. Zubov, On systems of ordinary differential equations with generalized homogeneous right-hand sides (in Russian), Izvestia vuzov. Mathematica, vol. 1, pp. 80-88, 1958.

[16] A. Bacciotti, L. Rosier, Lyapunov Functions and Stability in Control Theory, Springer, 2005.

[17] L. Rosier, Homogeneous Lyapunov function for homogeneous continuous vector field, Systems \& Control Letters, vol. 19, pp. 467-473, 1992.

[18] W.M. Haddad, A. L'Afflitto, Finite-time partial stability and stabilization, and optimal feedback control, Journal of the Franklin Institute, vol. 352, pp. 2329-2357, 2015.

[19] K.S. Narendra, A.M. Annaswamy, Stable adaptive systems, PrenticeHall, Inc. Upper Saddle River, NJ, 1989

[20] A. Polyakov, D. Efimov, W. Perruquetti, Finite-time and fixed-time stabilization: Implicit Lyapunov function approach, Automatica, vol. 51, pp. 332-340, 2015. 